\title{
THE RADIAL DISTRIBUTION OF THE KUIPER BELT
}

\author{
Chadwick A. Trujillo and Michael E. Brown \\ California Institute of Technology, MS 150-21, Pasadena, CA 91125; chad@gps.caltech.edu, mbrown@gps.caltech.edu \\ Received 2001 February 26; accepted 2001 May 11; published 2001 May 31
}

\begin{abstract}
We examine the radial distribution of the Kuiper Belt objects (KBOs) using a method that is insensitive to observational bias effects. This technique allows the use of the discovery distances of all KBOs, independent of orbital classification or discovery circumstance. We verify the presence of an outer edge to the Kuiper Belt, as reported in other works, and we measure this edge to be at $R=47 \pm 1$ AU given any physically plausible model of the size distribution. We confirm that this outer edge is due to the classical KBOs, the most numerically dominant observationally. In addition, we find that current surveys do not preclude the presence of a second, unobserved Kuiper Belt beyond $R=76 \mathrm{AU}$.
\end{abstract}

Subject headings: Kuiper Belt, Oort Cloud — minor planets, asteroids — solar system: formation

\section{INTRODUCTION}

The Kuiper Belt objects (KBOs) consist of a disk of icy bodies beyond Neptune's orbit, containing $\sim 0.06$ Earth masses of material (Trujillo, Jewitt, \& Luu 2001). The existence of an outer edge to the KBOs was first suggested by Dones (1997), who examined the detection statistics of the first six classical KBOs (eccentricities $e<0.15$ and semimajor axes $41 \mathrm{AU}<a<$ 47 AU) found by Jewitt, Luu, \& Chen (1996). Dones (1997) assumed a differential power-law size distribution (number of objects between radius $r$ and $r+d r$ following $\propto r^{-q} d r$ ) with exponent $q=3.5$. He found that the observations were in conflict with classical KBOs present beyond $50 \mathrm{AU}$ at the $98.5 \%$ confidence level. Jewitt, Luu, \& Trujillo (1998) reported a drop in the bias-corrected surface density of objects beyond $50 \mathrm{AU}$, using their best-fit $q=4$ size distribution. Gladman et al. (1998) argued that the size distribution measured by Jewitt et al. (1998) was too flat (had too few small objects). In the Gladman et al. (1998) best-fit $q=4.8$ model, the reduced discovery rates beyond $50 \mathrm{AU}$ were caused by the increased fraction of smaller objects, which were too faint to be detected beyond 50 AU. Chiang \& Brown (1999) measured a flatter $q=3.6$ size distribution and estimated that there is no KBO density enhancement beyond $50 \mathrm{AU}$ but could not effectively evaluate a density deficit with their sample. Trujillo et al. (2001) surveyed $73 \mathrm{deg}^{2}$ to red magnitude $m_{R}=23.7$ and found the strongest evidence to date for an outer edge to the classical KBOs using their new sample of objects. Allen, Bernstein, \& Malhotra (2001) also reported the presence of an outer edge to the Kuiper Belt at $R \sim 50 \mathrm{AU}$ in their survey of $1.5 \mathrm{deg}^{2}$ of sky to $m_{R} \sim 25.5$.

Currently, all works evaluating the presence of an edge have one or more of three basic deficiencies: (1) reliance on a limited subsample of objects, typically from surveys that the authors themselves have conducted, (2) edge distances not being explicitly evaluated, and (3) estimates being model dependent, with the assumed or fitted size distribution heavily influencing the final results. In this work, we present a simple method for determining the true radial distribution of the Kuiper Belt using all KBOs (a factor of 40 increase in sample size over previous works), regardless of discovery circumstances. We apply this method to the currently known sample of KBOs to measure the heliocentric distance of the outer edge. In addition, we place constraints on the existence of an as yet unobserved Kuiper Belt at large heliocentric distances.

\section{DATA USED}

All data used in this work are public domain, namely, the apparent visual magnitude $\left(m_{V}\right)$ and heliocentric distance $(R)$ of bodies upon discovery. This information can be obtained by computing the ephemerides of all bodies for the date and time of discovery given the orbital elements listed by the Minor Planet Center, as displayed in Figure 1. These two observational parameters are very reliable and subject to little change- $R$ is measured directly from an object's apparent parallactic motion, and $m_{V}$ is calibrated by the observer from standard stars. Of the 36 multiopposition bodies examined by Trujillo et al. (2001), none exhibited discovery $R$-changes of more than 0.5 AU between first and second opposition orbits. This differs from other descriptors, such as semimajor axis and eccentricity, which may take more observations to estimate. As one would expect, there is an apparent density deficit at large heliocentric distances, where KBOs are faintest. In the following sections, we estimate the intrinsic density versus heliocentric distance, corrected for observational biases against distant and faint objects.

\section{ANALYSIS}

The major assumption in our analysis is that detection of KBOs is biased by the brightness of the KBO but not by the radial distance of the object; i.e., a faint nearby object is detected with the same efficiency as a faint distant object. This is a reasonable assumption, because for a given apparent magnitude, there is only a single bias against discovering nearby objects compared to distant objects - the magnitude of parallactic motion. Most of the KBOs found in published surveys have had opposition parallax motion $1^{\prime \prime} \mathrm{hr}^{-1} \lesssim \dot{\theta} \lesssim 10^{\prime \prime} \mathrm{hr}^{-1}$, probing heliocentric distances $10 \mathrm{AU} \lesssim R \lesssim 140 \mathrm{AU}$ (Irwin, Tremaine, \& Żytkow 1995; Jewitt \& Luu 1995; Jewitt et al. 1996, 1998; Gladman et al. 1998; Luu \& Jewitt 1998; Trujillo \& Jewitt 1998; Chiang \& Brown 1999; Trujillo et al. 2001). One notable exception is Spacewatch, which is limited to objects moving faster than 2 "'25 $\mathrm{hr}^{-1}$ (Larsen et al. 2001).

We wish to derive the true heliocentric distance distribution of the KBOs, $f(R) d R$, representing the number of KBOs between heliocentric distances $R$ and $R+d R$. We derive this from the apparent distribution of objects, $f_{\text {app }}(R)$, where $f_{\text {app }}(R) d R$ is the number of objects observed with heliocentric distances between $R$ and $R+d R$. We assume a population with an albedo 


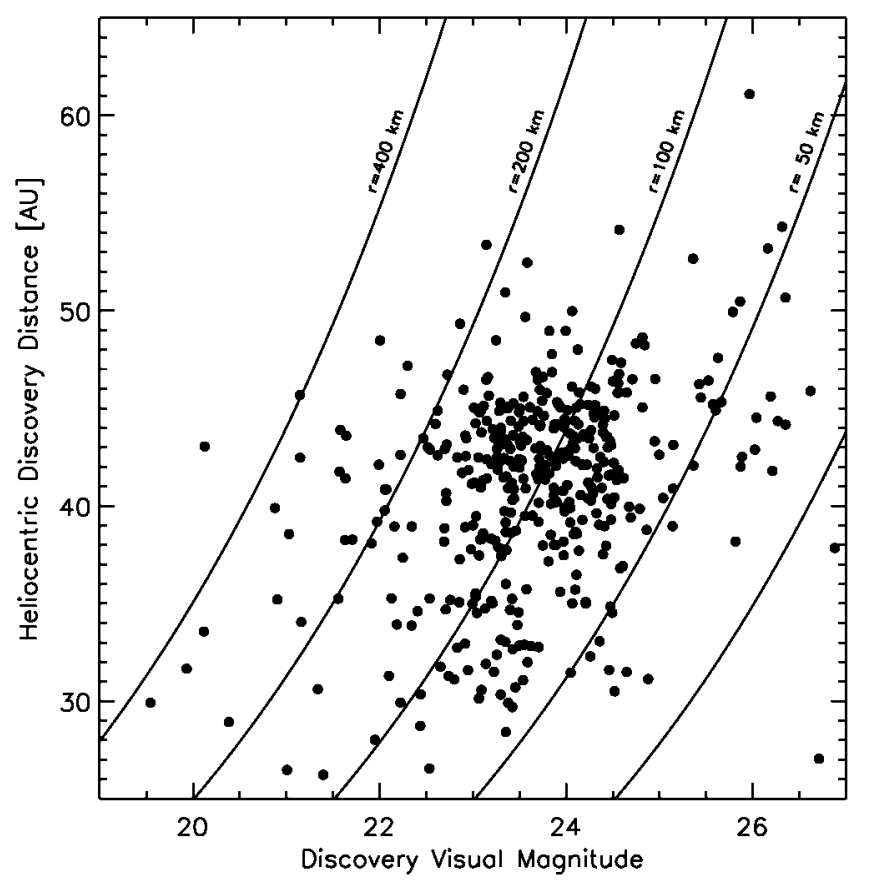

FIg. 1.-Heliocentric distance at discovery vs. visual magnitude at discovery; data are from the Minor Planet Center. Lines represent objects of constant radius, assuming a $4 \%$ albedo.

distribution independent of both size and heliocentric distance and a fixed differential size distribution with power-law index $q \neq 1$. The term $n(R, r) d R d r$ refers to the number of bodies with heliocentric distances between $R$ and $R+d R$ and radii between $r$ and $r+d r$,

$$
n(R, r) d R d r=\Gamma f(R) r^{-q} d R d r
$$

where $\Gamma$ is a normalization constant.

We would like to find the number of objects with apparent magnitudes between $m_{V}$ and $m_{V}+\Delta m_{V}$, corresponding to a radius range between $r_{0}$ and $r_{1}$ at any given heliocentric distance $R$ (in AU) and geocentric distance $\Delta$ (in AU):

$$
\begin{aligned}
& r_{0}=R \Delta 10^{0.2\left(m_{0}-m_{V}-\Delta m_{V}\right)}, \\
& r_{1}=R \Delta 10^{0.2\left(m_{0}-m_{V}\right)},
\end{aligned}
$$

given the constant

$$
m_{0}=m_{\odot}-2.5 \log p+2.5 \log \left(2.25 \times 10^{16}\right)
$$

derived from the formula for apparent visual magnitude,

$$
m_{V}=m_{\odot}-2.5 \log \left[p \Phi(\alpha) r^{2}\right]+2.5 \log \left(2.25 \times 10^{16} R^{2} \Delta^{2}\right) \text {. }
$$

Here $r$ is radius (in kilometers) and $p$ is the albedo. We assume opposition observations, such that the Sun-object-observer angle $\alpha \equiv 0$ and the object's phase function $\Phi(0) \equiv 1$. The apparent $V$ magnitude of the Sun is given by $m_{\odot}=-26.75$.

Opposition observations require $\Delta \equiv R-1$, and assuming an observing efficiency between magnitudes $m_{V}$ and $m_{V}+$ $\Delta m_{V}$ of $\epsilon\left(m_{V}\right)$, we integrate equation (1) over $r_{0}<r<r_{1}$, yield- ing the apparent heliocentric distance distribution:

$$
\begin{aligned}
f_{\mathrm{app}}(R) d R & =\epsilon\left(m_{V}\right) n(R) d R=\epsilon\left(m_{V}\right) \Gamma f(R) d R \int_{r_{0}}^{r_{1}} r^{-q} d r \\
& =\frac{\epsilon\left(m_{V}\right) \Gamma}{q-1} f(R) d R\left(r_{0}^{1-q}-r_{1}^{1-q}\right), \\
& =\Gamma^{\prime}\left(m_{V}\right) f(R)\left(R^{2}-R\right)^{1-q} d R
\end{aligned}
$$

where the magnitude-dependent terms have been absorbed into $\Gamma^{\prime}\left(m_{V}\right)$, given by

$$
\Gamma^{\prime}\left(m_{V}\right)=\epsilon\left(m_{V}\right) \frac{\Gamma}{q-1} 10^{0.2\left(m_{V}-m_{0}\right)(q-1)}\left(10^{0.2(q-1) \Delta m_{V}}-1\right) .
$$

Thus, the intrinsic heliocentric distance distribution $f(R) d R$ can be related to the apparent heliocentric distance distribution $f_{\text {app }}(R) d R$ for any given magnitude:

$$
f(R)=\Gamma^{\prime}\left(m_{V}\right)^{-1} \beta(R) f_{\mathrm{app}}(R),
$$

where the normalized bias-correction factor $\beta(R)=\left[\left(R^{2}-\right.\right.$ $\left.R) /\left(R_{0}^{2}-R_{0}\right)\right]^{q-1}$.

We do not compute the absolute normalization constant $\Gamma^{\prime}\left(m_{V}\right)$ because this would require a detailed study of all KBO survey efficiencies and sky areas examined. However, since the functional form of $f(R) d R$ does not vary with magnitude (i.e., the radial distribution does not depend on the magnitude of observation, as described earlier), the value of the parameter $\Gamma^{\prime}\left(m_{V}\right)$ can be calculated relative to $\Gamma^{\prime}$ for all other magnitudes by examining the numbers of objects discovered at each magnitude. Consequently, we can treat the estimate of $f(R)$ at any magnitude $m_{V}$ as an independent measurement. Summing all of these measurements results in our final estimate of $f(R)$, as described in $\S 4$. This does not allow an estimate of the total number of KBOs, simply the functional form of their radial distribution. Thus, the choice of $R_{0}$ is arbitrary; we use $R_{0} \equiv 43 \mathrm{AU}$.

The assumptions enabling this derivation are summarized as follows: (1) all KBOs follow the same size distribution, described by a differential power law, (2) observations are conducted at opposition allowing the transformation $\Delta=R-1$, and (3) the albedo is not a function of radius $r$ or heliocentric distance $R$. We contend that these three assumptions are reasonable. Numerous papers have been published estimating the size distribution of the KBOs, none of which have found evidence for a size distribution departing from assumption 1. Most surveys for distant bodies are conducted near opposition. For the 407 objects in our sample (as of 2001 May), only seven violated $\Delta=R-1$ by more than $0.5 \mathrm{AU}$, resulting in a radius correction of less than $3 \%$ from equations (2) and (3). Criterion 3 is the weakest criterion because to date we have little information about the KBO albedos. The albedo of the dark Centaurs has been measured to be $p \sim 4 \%$, and the KBOs are typically assumed to have this value (Jewitt \& Luu 2000), adopted here. This is in statistical agreement with Jewitt, Aussel, \& Evans (2001), who found the red albedo of (20000) Varuna to be $0.070_{-0.017}^{+0.030}$. Several trials were conducted using a spread of albedos, and no significant change to the results were observed (see $\S 4$ ). 


\section{RESULTS}

We plot the true heliocentric distance distribution $f(R)$ of the discovery data for a $q=4$ size distribution in Figure 2, with error bars representing the Poissonian $68 \%$ confidence limit on the apparent number of objects in each bin (Kraft, Burrows, $\&$ Nousek 1991). The presented values were created by binning the apparent heliocentric distance distribution to yield $f_{\text {app }}(R)$ and multiplying by $\beta(R)$ for each heliocentric distance bin to compute $f(R)$, as per equation (10). This assumes that all objects have a constant albedo, an assumption that is relaxed later in this section. This procedure implicitly scales each magnitude range by the number of objects in that magnitude range, so an explicit correction for the term $\Gamma^{\prime}\left(m_{V}\right)$ is not necessary. This technique is robust, as demonstrated by the fact that the data were divided into two groups, the faint half $\left(m_{V}>23.6\right)$ and the bright half, each yielding nearly identical results. The radial distribution of the faint bodies is slightly different from that of the bright bodies, offset by $\sim 1$ AU. This difference is very small, considering the huge magnitude range of bodies considered $\left(19<m_{V}<27\right)$ and the wide variety of surveys conducted. We therefore place the outer edge of the Kuiper Belt at heliocentric distance $R=47 \pm 1$ AU. This is consistent with other detections of a belt edge, with a stricter identification of edge location (Dones 1997; Jewitt et al. 1998; Allen et al. 2000; Trujillo et al. 2001).

Besides a drop in density, the deficit beyond $47 \mathrm{AU}$ can be explained only by violating our assumption that the apparent radial distribution is independent of magnitude. This can be done by introducing a physical change in the bodies beyond 47 AU. We present three such possibilities in order of increasing efficacy in explaining the results: (1) the slope of the size distribution $q$ may increase, (2) the maximum object size $r_{\max }$ may decrease, or (3) the albedo $p$ may be reduced. These changes must be abrupt and pronounced, explaining the factor of 6 drop in intrinsic object number between $R=43 \mathrm{AU}$ and $R=52$ AU.

In scenario 1 , we apply the $\Gamma^{\prime}\left(m_{V}\right)^{-1} \beta(R)$ correction from equation (10), using a steeper size distribution $q_{\text {split }}$ for $R>$ $R_{\text {split }}$. The lowest $q_{\text {split }}$ that results in a constant surface density occurring beyond $R_{\text {split }}$ occurs with an unphysically large $q_{\text {split }}>10$ with $R_{\text {split }}=44$ AU. Thus, the observations are very difficult to explain by an increased size distribution at distant heliocentric distances.

We estimate the maximum size needed to explain the low number of objects beyond $R=50$ AU by examining Figure 1 . It is apparent that only a very small maximum size would explain the observations, roughly $50 \mathrm{~km}<r_{\max }<100 \mathrm{~km}$. This is much smaller than the currently observed maximum size for $R<50 \mathrm{AU}, r_{\max } \sim 400 \mathrm{~km}$. The maximum growth size of the KBOs as a function of radial distance has not been well studied, although order-of-magnitude arguments based on the magnitude of the Keplerian shear in the region exist (Wetherill 1989). Thus, we can only state that for scenario 2 to cause the observed radial distribution, $r_{\max }$ must drop by a factor of $\sim 5$ in the $45 \mathrm{AU}<R<52 \mathrm{AU}$ region. Due to such abruptness, we find scenario 2 unlikely.

For scenario 3 to explain the drop, one must modify $p$ to change the $m_{0}$ term in $\Gamma^{\prime}\left(m_{V} \mid p\right)$, which represents the $\Gamma^{\prime}\left(m_{V}\right)$ computed using albedo $p$. We solve for our factor of 6 drop in observed density beyond 47 AU, $6=$ $\Gamma^{\prime}\left(m_{V} \mid p\right) / \Gamma^{\prime}\left(m_{V} \mid p^{\prime}\right)=\left(p / p^{\prime}\right)^{1.5}$, yielding a factor $p / p^{\prime}=3.3$ drop in albedo. Thus, we could explain the observed decrease in number beyond $47 \mathrm{AU}$ by an albedo change from $p=$

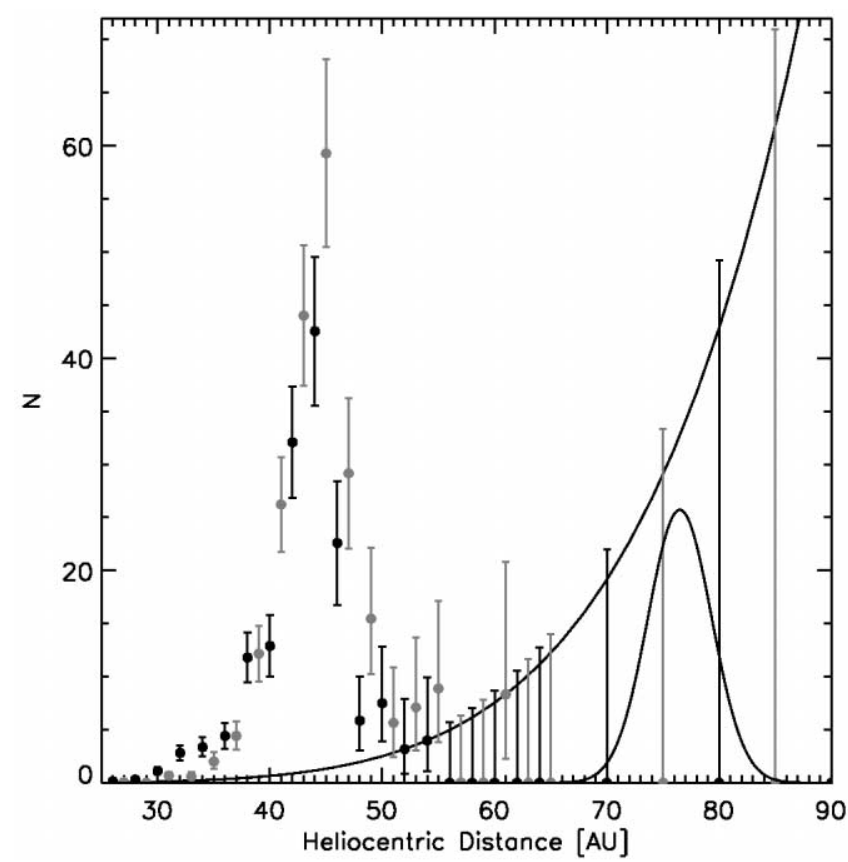

FIG. 2.-Filled circles: Binned intrinsic heliocentric distance distribution for a $q=4$ differential size distribution, with Poissonian error bars. The curve represents the correction $\beta(R)$ from apparent to intrinsic distributions. Gray values represent the faint half of the sample, while the black values represent the bright half of the sample. A pronounced drop in the density of objects is observed at $47 \mathrm{AU}$ in both cases. A subset of the data beyond $65 \mathrm{AU}$ have been plotted, for clarity. The Gaussian at $76 \mathrm{AU}$ represents the lower limit to the heliocentric distance of a second, unobserved Kuiper Belt (see $§ 5$ ).

0.13 (similar to Centaur Chiron; Campins et al. 1994) to $p^{\prime}=0.04$ (similar to the dark Centaurs; Jewitt \& Luu 2000) across the 45-52 AU region. Although these relative values of $p$ are physically plausible, the mechanism for confining lowalbedo objects to heliocentric distances $R>47 \mathrm{AU}$ is unknown, eliminating scenario 3 as an explanation for the observed outer edge.

All recent $\mathrm{KBO}$ survey results are roughly consistent with our $q=4$ size distribution. However, we have simulated the $q=3$ and $q=5$ cases in Figure 3, which both show the pronounced density drop at $47 \mathrm{AU}$. We have also conducted several trials to assess the constant albedo assumption of Figure 1 . In each trial, the discovery magnitude $m_{V}$ was modified by a random amount, drawn from a Gaussian with standard deviation $\sigma=2 \mathrm{mag}$, corresponding to a very large albedo change of a factor between 0.16 and $6.3(1 \sigma)$. The results of these trials did not vary significantly from those presented in Figure 2. Thus, we must conclude that the observed deficiency beyond $47 \mathrm{AU}$ is pronounced and real and is best explained by a drop in the number density of distant bodies.

In this analysis, no assumptions have been made about the orbital classification of objects: (1) the classical KBOs (semimajor axes $41 \mathrm{AU}<a<47 \mathrm{AU}$, eccentricities $e<0.15$ ), which comprise $70 \%$ of the observed objects; (2) the resonant KBOs, which are found in mean motion resonances with Neptune $(a \approx 39.4$ AU for the $3: 2$ "Plutinos" and $a \approx 47.7 \mathrm{AU}$ for the $2: 1$ ), representing about $20 \%$ of the observed sample; and (3) the scattered KBOs, which have large semimajor axes $(a>50 \mathrm{AU})$ and eccentric orbits $(e>0.3)$, which are about $10 \%$ of the total. Since we have only considered the observed ratios of the KBOs, our results are most applicable to the classical KBOs, which represent the largest portion of the known 


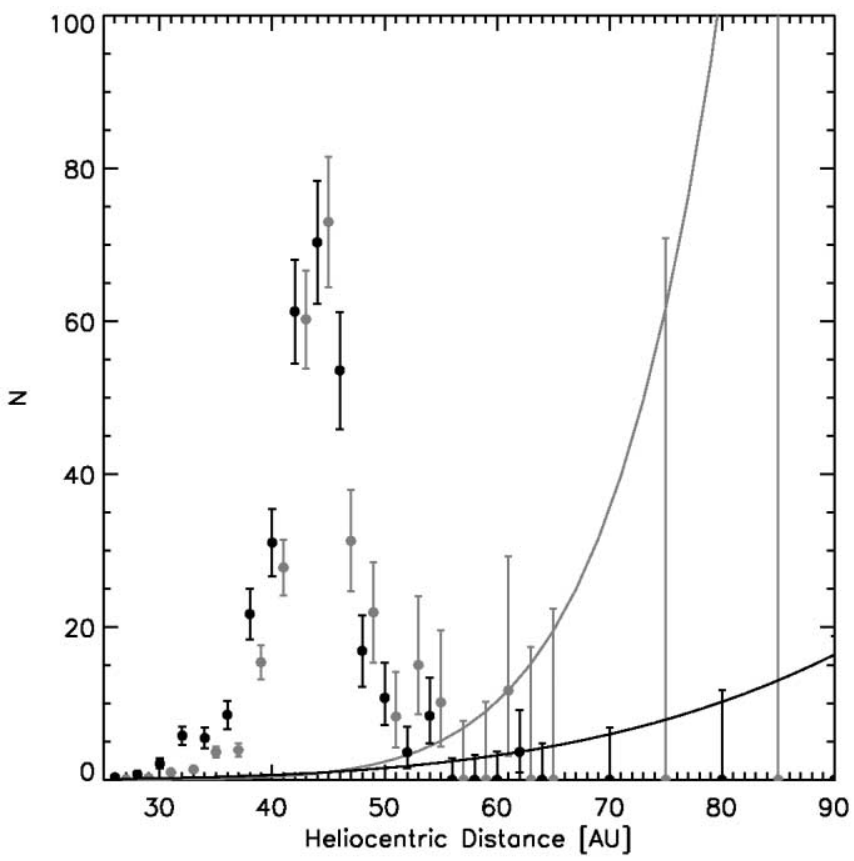

FIG. 3.-Filled circles: Intrinsic heliocentric distance distribution for $q=$ 3 (black) and $q=5$ (gray), with offset bin centers, for clarity. A pronounced drop in the density of objects is observed at $47 \mathrm{AU}$ in both cases. A subset of the points beyond $65 \mathrm{AU}$ have been plotted, for clarity.

sample. By confining our analysis to each of these populations in turn, we verify that the pronounced drop at $R=47 \pm 1 \mathrm{AU}$ is due to an outer edge to the classical KBO population, as reported by Trujillo et al. (2001).

\section{UNDETECTED POPULATION LIMITS}

Here we place constraints on the presence of a second Kuiper Belt, as yet undiscovered, at large heliocentric distances. We estimate a lower limit to the mean heliocentric distance of such a belt $\left(R_{\min }\right)$ by moving the known Kuiper Belt to extreme distances, assuming that the ecliptic plane surface density of KBOs is proportional to $R^{-2}$. We model the radial distribution of the undetected belt as a Gaussian with mean $\bar{R}$, amplitude proportional to $1 / \bar{R}$, and standard deviation $\sigma_{R}=3 \mathrm{AU}$, producing
$n_{i}(\bar{R})$ objects in the $i$ th bin located at heliocentric distance $R_{i}$. The Poisson probability that such a belt would remain unobserved is $P(\bar{R})=\prod_{i} \exp \left[-n_{i}(\bar{R}) / \beta\left(R_{i}\right)\right]$. The $3 \sigma(99.73 \%)$ lower limit to $R$ is computed by numerically solving the equation $P\left(R_{\text {min }}\right)=(1-0.9973)$, i.e., if $\bar{R}<R_{\min }$, such a belt would be detected in the observed data greater than $99.73 \%$ of the time. We find $R_{\min }=76$ AU from our model. Thus, a second, unobserved Kuiper Belt, if it exists, must have a mean heliocentric distance $\bar{R} \geq 76$ AU $(3 \sigma)$ in the $q=4$ model to be consistent with its null detection, as depicted in Figure 2.

This crude model relies on the fact that the brightest bodies in the undiscovered Kuiper Belt can be detected by most surveys. Excluding Pluto/Charon, the brightest KBO is 2000 $\mathrm{WR}_{106}$, with $m_{V}=20.1$ upon discovery at $R=43.0 \mathrm{AU}$. If $2000 \mathrm{WR}_{106}$ were moved to $R=76 \mathrm{AU}$, it would have $m_{V} \approx 22.6$, well within the range of most surveys. We do not consider the possibilities that such a distant belt may have a smaller maximum object size or substantial dynamical differences from the known KBOs, because there are no detailed dynamical models estimating such quantities. Thus, we report that a second, unobserved Kuiper Belt, if dynamically similar to the known Kuiper Belt, must have mean heliocentric distance $\bar{R} \geq 76$ AU.

\section{SUMMARY}

We have presented a robust technique for estimating the true radial distribution of the KBOs from the apparent distribution. No assumptions are made about the surveys conducted except that at any given magnitude, objects are found in a manner that is unbiased against proper motion. Applying this technique to all KBOs discovered in all surveys, we observe a pronounced density deficit occurring at heliocentric distance $R=47 \pm$ 1 AU for differential size distribution $q=4$, consistent with estimates of the belt edge by other researchers. This outer edge persists when considering size distributions $3<q<5$ and introducing substantial albedo variations. We confirm that this edge is due to the classical KBOs, the largest population of observed bodies in the Kuiper Belt. A second, unobserved classical belt with mean heliocentric distance $\bar{R}>76 \mathrm{AU}$ is also consistent with observations.

This project was funded by a NASA grant to M. E. B.

\section{REFERENCES}

Allen, R. L., Bernstein, G. M., \& Malhotra, R. 2001, ApJ, 549, L241

Campins, H., Telesco, C. M., Osip, D. J., Rieke, G. H., Rieke, M. J., \& Schulz, B. 1994, AJ, 108, 2318

Chiang, E. I., \& Brown, M. E. 1999, AJ, 118, 1411

Dones, L. 1997, in ASP Conf. Ser. 122, From Stardust to Planetesimals, ed.

Y. J. Pendleton \& A. G. G. M. Tielens (San Francisco: ASP), 347

Gladman, B., Kavelaars, J. J., Nicholson, P. D., Loredo, T. J., \& Burns, J. A. 1998, AJ, 116, 2042

Irwin, M., Tremaine, S., \& Żytkow, A. N. 1995, AJ, 110, 3082

Jewitt, D., Aussel, H., \& Evans, A. 2001, Nature, 411, 446

Jewitt, D. C., \& Luu, J. X. 1995, AJ, 109, 1867
Jewitt, D. C., \& Luu, J. X. 2000, in Protostars and Planets IV, ed. V. Mannings, A. P. Boss, \& S. S. Russell (Tucson: Univ. Arizona Press), 1201 Jewitt, D. C., Luu, J. X., \& Chen, J. 1996, AJ, 112, 1225

Jewitt, D. C., Luu, J. X., \& Trujillo, C. 1998, AJ, 115, 2125

Kraft, R., Burrows, D., \& Nousek, J. A. 1991, ApJ, 374, 344

Larsen, J. A., et al. 2001, AJ, 121, 562

Luu, J. X., \& Jewitt, D. C. 1998, ApJ, 502, L91

Trujillo, C., \& Jewitt, D. 1998, AJ, 115, 1680

Trujillo, C., Jewitt, D., \& Luu, J. 2001, AJ, in press

Wetherill, G. W. 1989, in The Formation and Evolution of Planetary Systems, ed. H. A. Weaver \& L. Danly (Cambridge: Cambridge Univ. Press), 1 